\title{
BALANCEO DE UN SISTEMA DE COSECHA MECANIZADO UTILIZANDO SIMULACIÓN DE EVENTOS DISCRETOS
}

\section{BALANCING A MECHANIZED HARVESTING SYSTEM USING DISCRETE EVENT SIMULATION}

\author{
Pablo Aracena ${ }^{1}$ Darío Aedo ${ }^{2}$ Ricardo Landeros ${ }^{3}$
}

\section{RESUMEN}

Se analizó un sistema de cosecha mecanizado, operando en una faena a tala rasa de pino radiata y conformado por un feller buncher, un skidder con garra, un procesador y un trineumático. Un Modelo de Simulación de Eventos Discretos (MSED) fue desarrollado con el propósito de balancear el sistema. El proceso mecanizado de madera fue el limitante del sistema de acuerdo con los resultados del estudio de tiempos, entonces este proceso fue apoyado agregando a 3 operadores de motosierra para alcanzar una producción de 60,82 $\mathrm{m}^{3} /$ Hora-Máquina (HM) en la configuración propuesta. Simulado el madereo se determinó que para balancear la producción obtenida en proceso, el skidder con garra debía operar a una distancia promedio de madereo (DPM) de $80 \mathrm{~m}$, realizando una detención para completar una carga de cuatro fustes. Finalmente, el feller buncher puede usar hasta 1,6 minutos adicionales para formar las pilas que permitan lograr la producción esperada para el sistema. De acuerdo con la experimentación de simulación se podría lograr una producción del sistema de $60,39 \mathrm{~m}^{3} / \mathrm{HM}$, si todas las modificaciones propuestas en volteo, apilado, madereo y proceso son realizadas.

Palabras-clave: simulación de eventos discretos; cosecha mecanizada; análisis de operaciones de cosecha.

\section{ABSTRACT}

A mechanized harvest system was analysed, working in a radiata pine clear cut operation and compound by a feller buncher, a grapple skidder, a grapple processor and a three-wheeler. A Discrete Event Simulation Model was built with the goal to balance the system. The mechanized wood processing was the bottleneck according to the time study results, then this process was supported adding 3 chain saw felling operators to reach a yield of $60.82 \mathrm{~m}^{3} /$ Productive Machine-Hour (PMH) in the configuration proposal. Also the simulated skidding was done, operating the grapple skidder at $80 \mathrm{~m}$ Average Skidding Distance (ASD) with one loading stop at a bunch for four stems to balance the obtained processing yield. Finally, the feller buncher can spend 1.6 extra minutes grouping the modify bunches to accomplish the expected yield of the system. The simulation experiment gives $60.39 \mathrm{~m}^{3} / \mathrm{PMH}$ expected yield of the system if all the proposed modifications in felling, bunching, skidding and processing are done.

Keywords: discrete event simulation; mechanized logging; harvesting operation analysis.

\section{INTRODUCCIÓN}

Dadas las exigencias productivas a que se ven enfrentadas las empresas de servicios forestales (emsefor), es necesaria una constante evaluación de los sistemas de aprovechamiento usados, de modo de detectar posibles fallas o funcionamientos no deseados y buscar potenciales soluciones. En este sentido, el tener un sistema productivo desbalanceado provoca inconvenientes de operación como: acumulación de materiales, máquinas subutilizadas, deficiencias en calidad de productos obtenidos, entre otros. Los sistemas de cosecha no escapan de esta problemática, por lo que es de interés mantener la operación de los mismos lo más fluida posible. Así el estudiar y tomar medidas de remediación en la operación de los mismos son actividades relevantes.

Los estudios de sistemas de operación pueden ser abordados con diferentes técnicas, entre las que se destacan los estudios de tiempos y rendimientos de campo, los modelos de simulación y los modelos de programación matemática. La selección de la técnica a usar depende principalmente de la variabilidad del sistema en estudio (AEDO, 1997). Es sabido que los sistemas de cosecha presentan alta variabilidad de

1. Ingeniero Forestal, Escuela de Ingeniería Forestal, Universidad Santo Tomás, Ejército 146, Santiago, Chile. paracena@santotomas.cl

2. Ingeniero Forestal, MSc., PhD., Departamento de Producción Forestal, Universidad de Talca, Casilla 747, Talca, Chile.daedo@utalca.cl

3. Ingeniero Forestal, Área Planificación de la Producción, Forestal Mininco, Casilla 399, Los Ángeles, Chile. ricardo.landeros@forestal.cmpc.cl

Recebido para publicação em 17/01/2006 e aceito em 18/04/2007. 
producción debido a los múltiples factores que los afectan, tales como: el estado del bosque, maquinarias que la componen, factores climáticos, productos a obtener, entre otros. Lo anterior hace aconsejable aplicar técnicas de análisis cuantitativo que permitan modelar la variabilidad real de los sistemas, con el propósito de obtener resultados lo más cercanos a esa realidad. Una técnica factible de aplicar en este caso es la Simulación de Eventos Discretos (SED) (JOHNSON, 1986). Wang y LeDoux (2003) la mencionan como una de las mejores técnicas para analizar operaciones de cosecha debido a la complejidad que presentan los sistemas de cosecha. De Toro y Hansson (2004a); De Toro y Hansson (2004b) han usado esta técnica con éxito en estudios sobre el uso de maquinaria agrícola para capturar la variabilidad de las condiciones climáticas durante diferentes años.

Randhawa et al. (1992) y Eliasson (1999) señalan que un modelo de simulación puede ser considerado como una versión de laboratorio del sistema con el cual es posible experimentar bajo condiciones controladas. Las condiciones de laboratorio posibilitan estudios difícilmente practicables en terreno por las condiciones del entorno, y recursos temporales y monetarios requeridos. Por ejemplo Aedo et al. (1998), en su investigación acerca de cómo afecta la forma del área de operación la productividad de un sistema de cosecha harvester - forwarder, aislaron en el modelo de simulación la variabilidad debida únicamente a esa característica geométrica; la experimentación es costosa y se torna difícil de llevar a cabo en terreno y por lo mismo impracticable. Así los investigadores mediante la experimentación con un MSED lograron estimar la influencia específica de la forma del área de operación sobre el rendimiento del sistema.

Otras investigaciones han usado modelos de simulación para seleccionar sistemas de cosecha adecuados a las condiciones de un rodal, dado que ellos afectan significativamente la eficiencia operacional de un sistema (Mc DONAGH et al., 2004).

En resumen, para que un MSED sea exitoso debe necesariamente incluir las características reales tanto del ambiente de operación (distancia de madereo, pendiente, topografía, etc.), del rodal (como densidad, volumen de productos, etc.), como también del sistema de cosecha (número y tipo de equipos, porcentajes de utilización, volúmenes madereados, etc.) (BAUMGRAS et al., 1993).

Algunos aspectos relevantes de ser considerados en el análisis de un sistema de cosecha por medio de un MSED son: la captura de datos de terreno, la obtención de datos de generación del modelo, la verificación del MSED generado, la determinación del periodo transitorio y número de corridas de experimentación, la factibilidad y facilidad de modificación de datos para la ejecución de una corrida experimental, y la experimentación misma con el modelo.

La captura de datos de terreno es la primera etapa y una de las más relevantes en la aplicación de un MSED, dado que contar con datos adecuados facilitará que los resultados obtenidos sean los correctos para la toma de decisiones. A partir de los datos de campo es posible obtener los datos de generación de un MSED, que corresponden a regresiones lineales y funciones de densidad de probabilidad, que modelan variables temporales y no temporales de la operación de un sistema en estudio. Estos datos de generación unidos a una lógica de operación del sistema modelado permiten la construcción de un potencial MSED de dicho sistema.

Previo al uso de un MSED debe considerarse su verificación; éste es un proceso mediante el cual los resultados arrojados por el modelo son contrastados con los datos de terreno. En esta etapa de la generación del MSED es posible detectar inconsistencias y corregirlas (ROSS, 1990 apud AEDO, 1994). Es así que Wang y LeDoux (2003) validan su modelo de simulación comparando las medias alcanzadas por variables operacionales del sistema con datos observados en terreno.

Otro aspecto a considerar en la construcción de un modelo de simulación es la determinación del periodo transitorio y número de iteraciones de experimentación. Autores como Law y Kelton (2000) y Aedo (1997) mencionan al periodo transitorio como la etapa inicial de la simulación, etapa donde aún no se ha alcanzado el estado estable de operación del sistema productivo. Al inicio de la simulación los datos obtenidos presentan una variabilidad no deseada debido a que el sistema inicia su operación vacío, sin entidades a través de él. Entonces se hace necesario calcular el tiempo adecuado que se debe dejar transcurrir antes de iniciar una toma de datos válidos para un posterior análisis. Además, una experimentación requiere determinar el número de iteraciones a considerar con tal de disminuir el error de estimación sin aumentar excesivamente el tiempo de ejecución. 
El presente estudio de balanceo usa datos capturados en un estudio previo de terreno, realizado en Forestal Mininco S.A., donde se analizó un sistema de cosecha mecanizado, formado por un feller buncher, un skidder con garra, un procesador y un trineumático. Los datos de terreno arrojaron un desbalanceo en la operación del sistema. Luego se persigue evaluar alternativas de balanceo del mismo por medio de la generación y experimentación con un MSED.

Las alternativas para balancear el sistema de cosecha se orientaron, en primer lugar, a aumentar el rendimiento del equipo con menor desempeño dentro del sistema (el procesador); luego a manejar las variables involucradas en la interacción del skidder con garra con el feller buncher; para finalmente determinar el rendimiento del sistema bajo diferentes distancias promedio de madereo.

\section{MATERIALES Y MÉTODO}

Los materiales requeridos para la realización del estudio fueron: programa estadístico Statgraphics; lenguaje de simulación ProModel PC, y datos de terreno del sistema de cosecha en análisis.

El sistema de cosecha operó en el fundo Colicheu, ubicado aproximadamente a 12 kilómetros al noroeste de la ciudad de Cabrero (Figura 1), en una faena a tala rasa. Las operaciones de cosecha se realizaron en un rodal de Pinus radiata D. Don con las características que se presentan en la Tabla 1. Cabe destacar que los análisis posteriores están referidos sólo a la operación del sistema en un frente de corte y no considera los traslados a diferentes rodales.

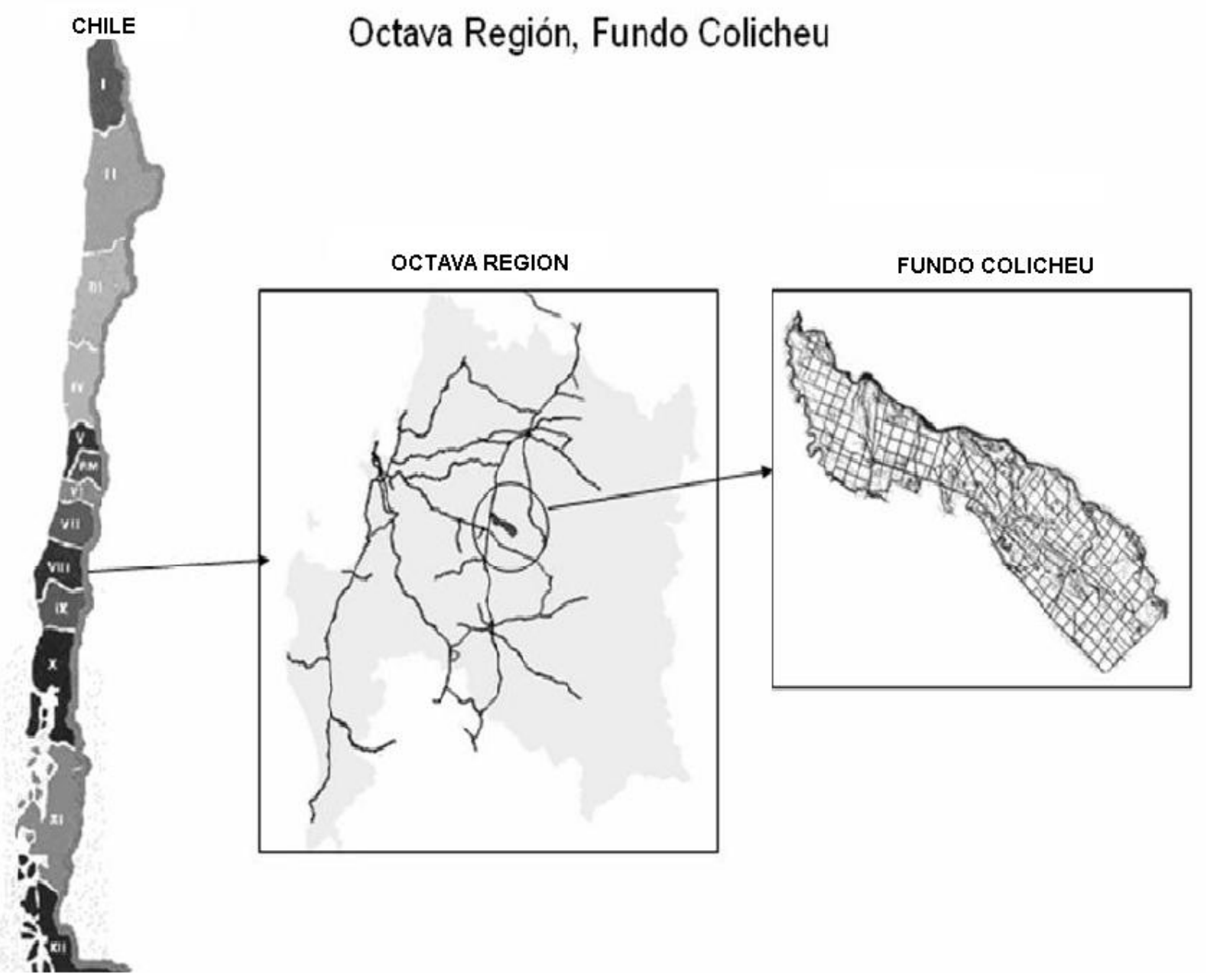

FIGURA 1: Ubicación del fundo Colicheu donde trabajó el sistema en estudio.

FIGURE 1: Colicheu farm location where the system under study worked. 
TABLA 1: Características del área de operación.

TABLE 1: Characteristics of the logging area.

\begin{tabular}{l|l}
\hline Variable & Descripción \\
\hline Superficie & 16,1 ha \\
Topografía & plana \\
Suelo & arena \\
Densidad & 462 árboles $/$ ha \\
Volumen por hectárea & $434,3 \mathrm{~m}^{3}$ \\
Volumen por árbol & $0,94 \mathrm{~m}^{3}$ \\
Manejo & dos raleos \\
\hline
\end{tabular}

Fuente: Ramírez (1997).

\section{Obtención de datos de generación del MSED}

Los datos de generación del MSED están constituidos por un conjunto de funciones de densidad de probabilidad y regresiones lineales. Éstas caracterizan tanto los productos que fluyen por el sistema (entidades), como las variables temporales y no temporales asociadas a la operación de cada una de las máquinas que conforman el sistema.

Existieron dos categorías de variables que requirieron del ajuste de funciones de distribución de probabilidad. La primera categoría la constituyó el diámetro a la altura del pecho, variable independiente que caracteriza a las entidades del sistema. La segunda categoría correspondió a variables temporales que caracterizan los tiempos operacionales de las máquinas, que carecen de una regresión lineal aceptable (tiempo de desplazamiento y tiempo de aproximación y limpieza del feller buncher; el tiempo de carga y descarga del skidder con garra; y el tiempo de preparación del procesador). Los datos se ajustaron a un conjunto de distribuciones teóricas, para cada una se obtuvo el valor-p de aplicar la prueba de bondad de ajuste Kolmogorov-Smirnov (KS) y se eligió para la modelación de cada variable analizada, la distribución que entregaba el mayor valor-p. Mientras mayor es el valor-p, existe una menor evidencia para rechazar la hipótesis de que el conjunto de datos observados es una muestra independiente de una función de distribución específica (LAW y KELTON, 2000).

El uso de regresiones lineales fue necesario debido a la dependencia que presentaron algunas variables temporales respecto de otras del sistema. Por ejemplo, el tiempo de volteo del feller buncher depende del diámetro a la altura del pecho (DAP), o el tiempo de operación del procesador que depende del diámetro basal y el tipo de rama que presentaban los árboles. Las regresiones lineales se ajustaron con el método de los mínimos cuadrados, además cumplieron con los supuestos de normalidad, homocedasticidad y no multicolinealidad. Además, se probó la significancia de los coeficientes de regresión. Comprobados estos

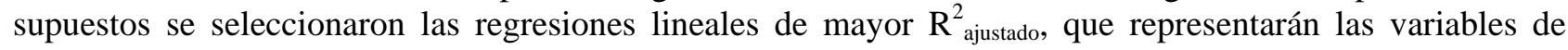
interés.

Entonces, conocida la distribución de modelación del DAP y la regresión lineal que lo relaciona, por ejemplo, con el tiempo de volteo, la simulación de diferentes situaciones se logró con la generación aleatoria de diversos DAP para representar diferentes árboles, obteniéndose tiempos de volteo diferentes y consistentes con las dimensiones de los árboles. El proceso se ejecuta primero generando un DAP aleatoriamente (de acuerdo con su distribución de modelación), este DAP, que corresponde a un árbol en particular, alimenta una regresión lineal como variable independiente la que arroja un valor esperado para un tiempo del proceso, como puede ser el tiempo de volteo. Este proceso se repite durante el tiempo que dura la simulación.

\section{Generación, verificación y dimensionamiento del MSED}

Una vez representadas las variables de interés del sistema y del bosque a través de funciones de distribución de probabilidad y de regresiones lineales, se implementó el MSED en el lenguaje de simulación ProModel PC. La construcción del modelo en dicho lenguaje consideró la definición de estructuras (localizaciones, entidades y recursos), procesos (proceso lógico, procesos de generación de entidades) y variables globales.

Junto con la construcción del modelo se representaron gráficamente localizaciones, entidades y 
recursos del sistema con el propósito de realizar una animación del mismo apoyado por una librería gráfica disponible en ambiente ProModel PC.

Cabe destacar que el trineumático fue eliminado del análisis debido a las siguientes razones: el rendimiento potencial de este equipo es un 93,4\% mayor al rendimiento del sistema; en los resultados preliminares entregados por el MSED, el trineumático presentó un tiempo ocioso del 91,1\%; el valor de adquisición y el costo de operación del trineumático es más bajo comparado con las otras máquinas; y al eliminar este equipo del modelo se redujo considerablemente el tiempo de computador requerido para realizar la experimentación.

\section{Verificación y Determinaciones de Experimentación}

Un MSED no debe ser usado en experimentación sin previamente determinar la consistencia de sus resultados con los reales. La verificación consistió en realizar corridas de prueba, obteniéndose datos de salida para la correspondiente comparación con los datos reales de terreno. Así fueron corregidas inconsistencias que presentó el modelo para ajustar sus resultados a los datos reales.

Para determinar el periodo transitorio se graficó la producción horaria del sistema en función del tiempo, en las etapas iniciales de la experimentación la variabilidad no corresponde a la real del sistema ya que el rendimiento se afecta por las condiciones iniciales, efecto que disminuye en la medida que aumenta el tiempo de experimentación.

Una vez determinado el periodo transitorio se calculó el número de iteraciones de experimentación considerando que los resultados se expresarían como un intervalo de confianza para la media y asumiendo que el resultado de cada corrida es independiente e idénticamente distribuido. Para el cálculo del número de iteraciones se realizaron un número fijo de corridas a modo de muestreo, considerando que la experimentación es un diseño al azar con población infinita y un error de experimentación menor al 5\%.

\section{RESULTADOS Y DISCUSIÓN}

Una vez construido el modelo de simulación se verificó y determinó un periodo transitorio de 40 horas y 30 iteraciones de experimentación de balanceo para obtener un error inferior a 5\%. El contar con un modelo representativo del sistema real permite la evaluación de las alternativas de operación del mismo.

\section{Experimentación de balanceo}

Considerando que el sistema original estaba desbalanceado, el procesador era la máquina crítica pues limitaba la producción del sistema en su conjunto al procesar sólo 41,23 m³/HM (ver Tabla 2). De acuerdo con la evaluación realizada en el estudio de tiempos y rendimientos se determinó que una de las variables que afectaba el tiempo de proceso fue el tipo de ramas (delgadas o gruesas) de los árboles. Por lo tanto, se consideró introducir motosierristas para apoyar la labor de procesamiento de árboles de ramas gruesas. Dado lo anterior y considerando la alta productividad que presentaba el feller buncher, se simuló además la operación del mismo apilando árboles pues, la operación original no consideraba este apilamiento. De esta manera, el feller buncher proveía una carga completa al skidder con garra, evitando detenciones de precarga a la máquina de madereo.

TABLA 2: Resultados del MSED en base a datos originales.

TABLE 2: MSED results based on field data.

\begin{tabular}{l|c|c|c}
\hline Equipo & $\begin{array}{c}\text { Rendimiento Promedio } \\
\left(\mathrm{m}^{3} / \mathrm{HM}\right)\end{array}$ & $\begin{array}{c}\text { Desviación Estándar } \\
\left(\mathrm{m}^{3} / \mathrm{HM}\right)\end{array}$ & $\begin{array}{c}\text { IC } \\
\left(\mathrm{m}^{3} / \mathrm{HM}\right)\end{array}$ \\
\hline Feller buncher & 140,17 & 1,50 & $139,61-140,73$ \\
Skidder con garra & 51,58 & 1,64 & $50,96-52,19$ \\
Procesador & 41,23 & 0,58 & $41,02-41,45$ \\
\hline
\end{tabular}

En que: IC = Intervalo de confianza al 95\%.

Las gavillas debían ser de un tamaño que permitiera al skidder con garra aumentar su rendimiento. Por lo tanto, en primer lugar se determinó el tamaño de carga que producía un mayor rendimiento del skidder. Luego, se determinó el tiempo de apilado máximo del feller buncher para evaluar la factibilidad de esta modificación. Finalmente, dadas las modificaciones anteriormente propuestas y de modo de obtener un sistema balanceado, fue necesario determinar la dependencia del rendimiento del skidder con garra de la 
DPM de operación.

El balance se ejecutó en dos etapas. Primero, se trabajó sobre las variables que afectan el rendimiento del procesador, equipo que presenta la más baja producción horaria. Una vez que el procesador dejó de ser el cuello de botella, se trabajó sobre las variables que afectan el rendimiento del skidder pues, este equipo pasó a limitar productivamente el sistema.

Cabe destacar las bajas desviaciones estándar obtenidas con 30 iteraciones (Tabla 2), lo que genera errores de muestreo inferiores a 1,2\%. La obtención de valores pequeños se debe a factores como: la homogeneidad del bosque, terreno plano y cortas distancias de madereo; condiciones de operación que son propicias para el desempeño homogéneo de los equipos de cosecha.

\section{Disminución del tiempo de procesamiento}

El tiempo de procesamiento de la madera fue disminuido considerando dos modificaciones a la operación original: 1) agregar motosierristas para apoyar al procesador y 2) dejar que éste último procese sólo árboles de ramas delgadas. Para evaluar la modificación anterior, se determinó el rendimiento del procesador simulando diferentes porcentajes de árboles de ramas delgadas en el sistema (Tabla 3).

TABLA 3: Producción del procesador de acuerdo al porcentaje de árboles de ramas delgadas.

TABLE 3: Processor yield according to percentage of trees with thin branches.

\begin{tabular}{cccccc}
\hline $\mathrm{ARD}(\%)$ & $\mathrm{RP}\left(\mathrm{m}^{3} / \mathrm{HM}\right)$ & $\mathrm{DE}\left(\mathrm{m}^{3} / \mathrm{HM}\right)$ & $\mathrm{IC}\left(\mathrm{m}^{3} / \mathrm{HM}\right)$ & $\mathrm{VR}(\%)$ \\
\hline 0 & 37,23 & 0,49 & $37,05-37,41$ & $-9,7$ \\
50 & 39,92 & 0,76 & $39,64-40,21$ & $-3,2$ \\
75 & 41,23 & 0,58 & $41,02-41,45$ & 0,0 \\
100 & 42,82 & 0,72 & $42,55-43,08$ & $+3,9$ \\
\hline
\end{tabular}

En que: $\mathrm{ARD}$ = Árboles de ramas delgadas; RP = Rendimiento promedio; DE = Desviación estándar; IC = Intervalo de confianza al 95\%; VR = Variación en rendimiento con respecto a la operación original.

Se observa una relación directamente proporcional entre el rendimiento promedio y el porcentaje de árboles de ramas delgadas en el sistema, es decir, mientras mayor es la proporción de árboles de ramas delgadas que son procesados menor es el tiempo que demora el procesador en realizar su faena.

El mínimo rendimiento del procesador se produce cuando el porcentaje de árboles de ramas delgadas en el sistema es cero, es decir, cuando procesa sólo árboles de ramas gruesas. En el otro extremo, se produce el máximo rendimiento cuando procesa solamente árboles de ramas delgadas (Figura 2).

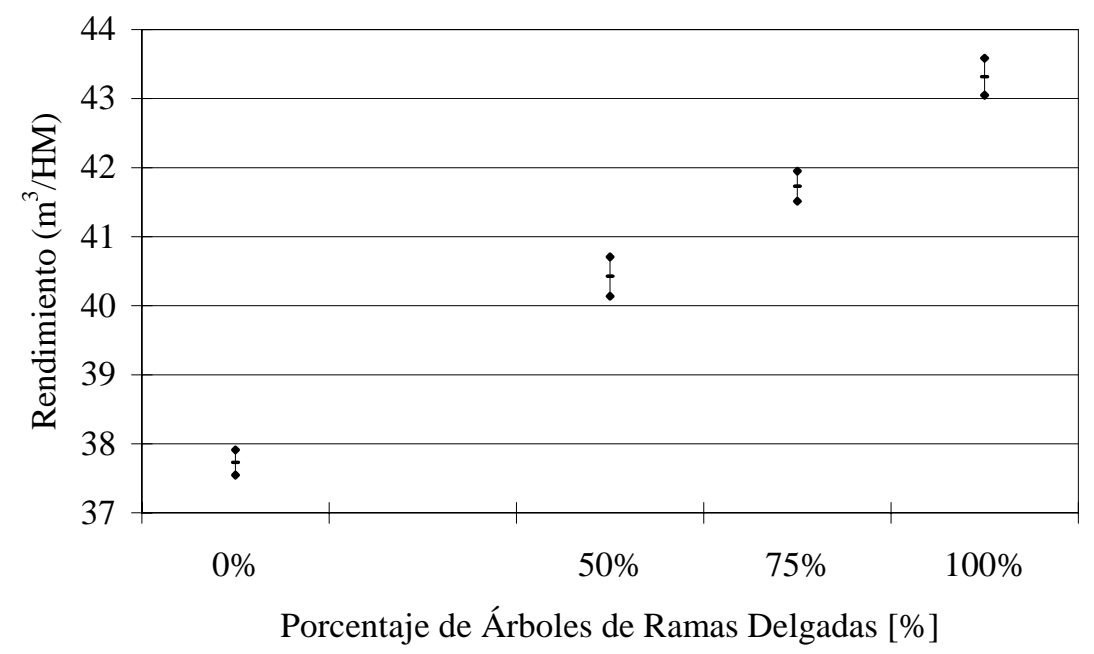

FIGURA 2: Producción horaria en función del porcentaje de árboles de ramas delgadas.

FIGURE 2: Hourly yield based on the percentage of trees with thin branches.

Si se incorporaran motosierristas al procesamiento de árboles de ramas gruesas, la producción horaria del sistema aumentaría. Además del rendimiento promedio del procesador, asumiendo que procesa el $100 \%$ de árboles de ramas delgadas $\left(42,82 \mathrm{~m}^{3} / \mathrm{HM}\right)$, se debería agregar el rendimiento de motosierristas 
procesando árboles de ramas gruesas. Por el contrario, si no se agregaran motosierristas al sistema, la experimentación de balanceo terminaría en este punto, lográndose sólo un incremento de 3,86\% en la producción horaria del sistema. Además, los árboles considerados de ramas gruesas se acumularían, ya que no serían procesados.

Si se considera que un motosierrista procesa en promedio $6 \mathrm{~m}^{3} / \mathrm{HM}$ (un valor pesimista de acuerdo con estándares nacionales para bosques de Pinus radiata de características similares a las del estudio), tres de ellos deben apoyar al procesador para lograr un rendimiento promedio de 60,82 $\mathrm{m}^{3} / \mathrm{HM}$ aproximadamente, así el subsistema de procesamiento dejaría de ser limitante. En esta situación quedaría el skidder con garra, con un rendimiento de 51,58 $\mathrm{m}^{3} / \mathrm{HM}$. Por lo tanto, bajo estas condiciones es necesario trabajar tanto con el feller buncher como con el skidder con garra para balancear el sistema.

Este resultado puede ser importante al realizar la planificación de la cosecha, con el propósito de lograr mayores rendimientos promedio, en dos aspectos: 1) considerar el tipo de manejo del bosque al que se asigne los diferentes sistemas de cosecha; y 2) considerar el apoyo del procesamiento mecanizado de madera a través de faenas tradicionales.

Se debe considerar que aplicar esta modificación en terreno implica una clasificación de los árboles (previa al procesamiento) separándolos en dos tipos: ramas delgadas y gruesas. Es factible que esta actividad la realice el trineumático, en cuyo operador recaería la responsabilidad de la clasificación.

\section{Aumento del rendimiento en madereo}

Los pasos a seguir en esta etapa son: 1) determinar el número de árboles por carga con el cual se obtenga el mayor rendimiento para la máquina de madereo; 2) determinar el tiempo máximo que puede destinar el feller buncher a realizar la faena de apilado; y 3) determinar el rendimiento bajo diferentes DPM.

De acuerdo con los datos de terreno, el skidder generalmente transportaba 3 ó 4 árboles por carga (cuando realizaba una detención). Por lo tanto, se determinó la producción horaria del skidder para ambos tamaños de carga (considerando que operaba a los 90 m de DPM de la operación muestreada). Los resultados de rendimiento promedio, desviación estándar e intervalos de confianza al 95\% para los diferentes tamaños de carga se presentan en la Tabla 4.

TABLA 4: Producción horaria del skidder con garra bajo diferentes tamaños de carga.

TABLE 4: Hourly yield of grapple skidder under different load sizes.

\begin{tabular}{cccccc}
\hline $\mathrm{N}$. & $\mathrm{RP}\left(\mathrm{m}^{3} / \mathrm{HM}\right)$ & $\mathrm{DE}\left(\mathrm{m}^{3} / \mathrm{HM}\right)$ & $\mathrm{IC}\left(\mathrm{m}^{3} / \mathrm{HM}\right)$ & $\mathrm{VR}(\%)$ \\
\hline 3 & 45,92 & 1,57 & $45,33-46,50$ & $-11,0$ \\
4 & 57,70 & 1,93 & $56,98-58,42$ & $+11,9$ \\
\hline
\end{tabular}

En que: $\mathrm{N}=\mathrm{N}$. Árboles por Carga; RP = Rendimiento Promedio; DE = Desviación Estándar; IC = Intervalo de Confianza al 95\%; VR = Variación en Rendimiento con Respecto a la Operación Original.

Como se observa en la Tabla 4, el tamaño de carga con el que se logró un mayor rendimiento fue de 4 árboles $\left(57,70 \mathrm{~m}^{3} / \mathrm{HM}\right)$. En la Figura 3 se observa la diferencia en rendimiento entre llevar 3 o 4 árboles por carga. Por lo tanto, el tamaño conveniente de pilas que debería formar el feller buncher para el skidder deberían ser de 4 árboles. Es importante destacar que con sólo realizar esta modificación (del tamaño de carga), se podría lograr un incremento de $6,12 \mathrm{~m}^{3} / \mathrm{HM}$ (desde $51,58 \mathrm{~m}^{3} / \mathrm{HM}$ a $57,70 \mathrm{~m}^{3} / \mathrm{HM}$ ) en el rendimiento del skidder. 


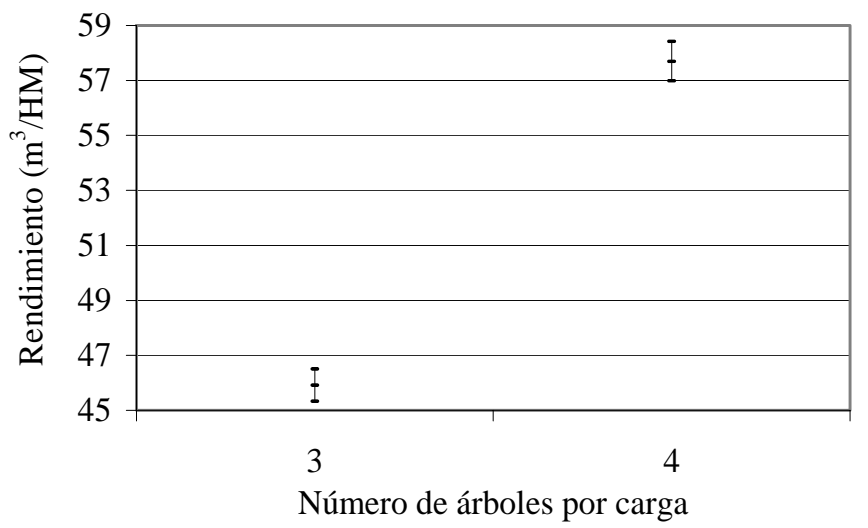

FIGURA 3: Producción horaria del skidder con garra bajo diferentes tamaños de carga.

FIGURE 3: Hourly yield of grapple skidder under different load sizes.

En el punto anterior se determinó que apilando 4 árboles el skidder con garra alcanzaría su mayor rendimiento (considerando sólo esta variable). En este punto es de interés conocer cuánto tiempo puede destinar como máximo el feller buncher para realizar pilas de 4 árboles, de modo que este último no se torne limitante para el sistema.

Debido a la falta de datos de terreno con respecto a tiempos de apilado se evaluó el rendimiento del feller buncher tomando como referencia el tiempo de volteo del mismo. Los resultados de esta experimentación se presentan en la Tabla 5.

TABLA 5: Rendimiento del feller buncher para diferentes tiempos de apilado.

TABLE 5: Feller-Buncher yield for different bunching times.

\begin{tabular}{ccccc}
\hline TA* & $\mathrm{RP}\left(\mathrm{m}^{3} / \mathrm{HM}\right)$ & $\mathrm{DE}\left(\mathrm{m}^{3} / \mathrm{HM}\right)$ & $\mathrm{IC}\left(\mathrm{m}^{3} / \mathrm{HM}\right)$ & Valor-p $\mathrm{p}^{* *}$ \\
\hline 0,0 & 140,17 & 1,50 & $139,61-140,73$ & $<0,0001$ \\
1,0 & 95,61 & 1,12 & $95,19-96,03$ & $<0,0001$ \\
2,0 & 71,97 & 0,98 & $71,60-72,33$ & $<0,0001$ \\
2,5 & 64,26 & 0,79 & $63,97-64,56$ & $<0,0001$ \\
2,7 & 61,39 & 0,83 & $61,07-61,70$ & $<0,0001$ \\
2,8 & 60,30 & 0,71 & $60,04-60,57$ & $<0,0001$ \\
2,9 & 59,19 & 0,79 & $58,90-59,49$ & 0,0002 \\
3,0 & 58,10 & 0,83 & $57,79-58,41$ & 0,2968 \\
\hline
\end{tabular}

En que: TA = Tiempo de apilado (N. de veces el tiempo de volteo); RP = Rendimiento promedio; DE = Desviación estándar; IC = Intervalo de confianza al 95\%; * = el tiempo de volteo promedio es igual a 8,8 segundos; ** = resultado de una prueba t, que corresponde a la comparación del rendimiento promedio del feller buncher con respecto al rendimiento promedio del skidder con garra [con 4 árboles por carga $\left.\left(57,70 \mathrm{~m}^{3} / \mathrm{HM}\right)\right]$.

Para tiempos de apilado de hasta 2,9 veces el tiempo de volteo, el rendimiento del feller buncher sigue siendo mayor que el del skidder (valores-p menores a 0,0002). Por lo tanto, el rendimiento del feller buncher no es limitante para el sistema. Por otro lado, cuando el tiempo de apilado es 3 veces el tiempo de volteo, el rendimiento del feller buncher es menor que el del skidder (valor-p $=0,2968$ ). En resumen, el feller buncher podría destinar hasta 25,5 segundos por árbol para realizar la faena de apilado. Por consiguiente, hasta 102 segundos extra podría el feller buncher destinar a completar una pila de 4 árboles sin que esto afecte la producción horaria de él o del sistema.

Estos resultados pueden ser de importancia al momento de la planificación operativa de la cosecha. $\mathrm{Al}$ realizar estas modificaciones de fácil aplicación operacional y de bajo costo, se podría lograr un aumento significativo en el rendimiento del sistema.

Anteriormente se estableció que sería totalmente factible que el feller buncher realizara pilas de 4 árboles para el skidder. Pero no fue suficiente el incremento del rendimiento logrado por este último equipo para balancear el sistema $\left(50,57 \mathrm{~m}^{3} / \mathrm{HM}\right)$, puesto que la faena de procesamiento alcanzó un rendimiento de 
60,82 $\mathrm{m}^{3} / \mathrm{HM}$ (considerando 3 motosierristas apoyando al procesador). Por lo tanto, se pretende encontrar la DPM en la que se logre balancear el sistema.

Con el propósito de generar una regresión de rendimiento en función de la DPM y a la vez determinar la DPM que permita alcanzar el rendimiento en proceso, se simuló la operación del skidder con garra bajo diferentes DPM alternativas (de 70 a $110 \mathrm{~m}$ ). Se realizaron cinco corridas (una para cada DPM), de 30 iteraciones cada una. Así se obtuvieron 30 datos de producción horaria para cada valor de DPM analizada. La regresión lineal que se ajustó entre el rendimiento $\left(\mathrm{m}^{3} / \mathrm{HM}\right)$ y la DPM (m) se presenta a continuación:

$$
\begin{array}{ccc}
\operatorname{Med}(\text { Rendimiento } \mid D P M) & =88,248448 & -0,348198 * D P M \\
\text { Valor }-p & <0,0001 & 0,0001
\end{array}
$$

En que: $\mathrm{R}_{\text {ajustado }}^{2}=0,9110 ; 70 \leq \mathrm{DPM} \leq 110 ; \mathrm{n}=150$

Esta regresión lineal, al igual que las usadas como datos de generación del modelo, cumplió con los supuestos de normalidad y homocedasticidad de una regresión.

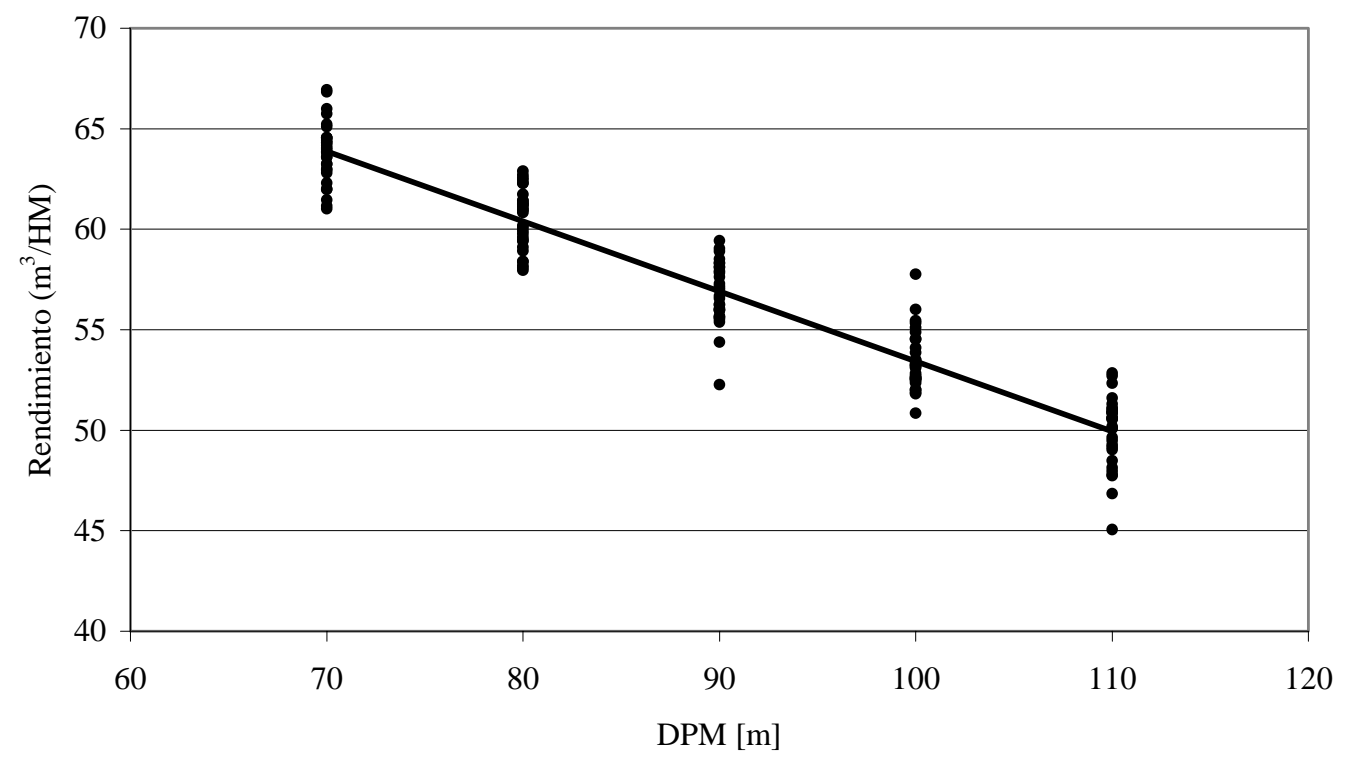

FIGURA 4: Producción horaria para diferentes DPM.

FIGURE 4: Hourly yield for different DPM.

$\mathrm{Al}$ analizar la Figura 4 y aplicando punto de equilibrio se desprende que para balancear el sistema se requeriría que el skidder trabajara a $80 \mathrm{~m}$ de DPM aproximadamente para así lograr $60,8 \mathrm{~m}^{3} / \mathrm{HM}$ de rendimiento del skidder y del sistema.

Sin embargo, el tiempo de apilado máximo del feller buncher cambió en este caso (DPM $=80 \mathrm{~m})$ a 2,7 veces el tiempo de volteo. Es decir, 1,6 minutos en realizar una pila de 4 árboles (6 segundos menos de tiempo que para una DPM de $90 \mathrm{~m}$ ).

Cabe destacar que las distancias promedio de madereo de la magnitud presentadas en este estudio pueden ser consideradas pequeñas, comparadas con otras operaciones del sistema de alta producción, esto redundará en altas densidades camineras y costos elevados de construcción de caminos. Aún así, la DPM de operación es una característica del sistema, lo que no constituye una limitante a la aplicación de SED en el análisis de balanceo de la producción del mismo.

\section{CONCLUSIONES}

En resumen, la línea de producción quedaría balanceada si: 1) el feller buncher genera pilas de 4 árboles gastando un tiempo de hasta 1,6 minutos, con lo que su rendimiento bajará, pero no se tornará limitante para el sistema; 2) el skidder con garra realiza una detención para cargar una pila de 4 árboles en 
cada ciclo, operando a $80 \mathrm{~m}$ de DPM; y 3) el procesamiento se realiza por un procesador $\left(42,82 \mathrm{~m}^{3} / \mathrm{HM}\right)$ y 3 motosierristas con rendimiento promedio de $18 \mathrm{~m}^{3} / \mathrm{HM}$, que da un total de $60,82 \mathrm{~m}^{3} / \mathrm{HM}$. De esta manera, el sistema tendría un rendimiento promedio de $60,39 \mathrm{~m}^{3} / \mathrm{HM}$, valor que equivale a un incremento de $46 \%$ con respecto al rendimiento del sistema en las condiciones de operación originales.

La aplicación de SED para evaluar las alternativas de operación del sistema de cosecha en estudio se presenta como una técnica conveniente pués, permitió analizar una variedad de alternativas de operación que son impracticables de llevar a cabo en terreno por el costo que involucraría. Además, experimentando en el computador fue posible simular la operación del sistema en tiempo reducido, una hora de simulación fue equivalente a 30 jornadas de operación en tiempo real. Por otro lado, implementar en el computador las modificaciones de operación del sistema no representa riesgo de accidente para los operadores, a diferencia de si se realizaran al sistema real. Estos aspectos hacen de SED una técnica eficiente en el estudio operacional de sistemas de cosecha, evaluando alternativas de operación bajo situaciones de laboratorio y sin olvidar que la definición final de cualquier modificación propuesta al sistema dependerá de la operación en terreno del sistema real.

\section{REFERENCIAS BIBLIOGRÁFICAS}

AEDO, D.; NEUENSCHWANDER, R.; ARACENA, P. Harvesting yield related to geometric form of the operation area. In: COFE proceedings: Harvesting logistics: from wood to markets. Portland, Oregon, Estados Unidos: 1998. p.53-53.

AEDO, D. Simulación de producción forestal: ¿̇epresentación de la realidad? In: Sistemas de Producción Forestal: análisis, cuantificación y sustentabilidad. Talca, Chile: Departamento de Producción Forestal. Facultad de Ciencias Forestales. Universidad de Talca: 1997. p.103-112.

AEDO, D., OLSEN, E.D.; KELLOGG, L. Simulating a harvester-forwarder softwood thinning: a software evaluation. Forest Products Journal, v. 47, n.5, p. 36-41, 1997.

AEDO, D. A mechanized harvesting system simulation: input, output, limitation and capabilities. 1994. $109 \mathrm{f}$. Tesis (Master of Science) - Oregon State University. Corvallis, Estados Unidos, 1994.

BAUMGRAS, J., HASSLER, C.; LEDOUX, C. Estimating and validating harvesting system production through computer simulation. Forest Products Journal, v. 43, n. 11/12, p. 65 - 71, 1993.

DE TORO, A.; HANSSON, A. Analysis of field machinery performance based on daily soil workability status using discrete event simulation or on average workday probability. Agricultural Systems, v. 79, n. 1, p. 109-129, 2004a.

DE TORO, A.; HANSSON, A. Machinery Co-operatives - a Case Study in Sweden. Biosystems Engineering, v. 87, n. 1, p. 13-25, 2004b.

ELIASSON, L. Simulation of thinning with a single-grip harvester. Forest Science, v. 45, n. 1, p. 26 - 34, 1999.

JOHNSON, L.R. Perspectives on the application of simulation to industrial forestry. In: Convención Nacional del SAF. Alabama, Estados Unidos: 1986. 4p.

LAW, A.; KELTON, W. Simulation modeling and analysis. 3. ed. New York: McGraw Hill, Inc, 2000. 760 p.

MC DONAGH, K,; MELLER, R.; VISSER, R. et al. Harvesting system simulation using a systems dynamic model. Southern Journal of Applied Forestry, v. 28, n. 2, p. 91 - 99, 2004.

RAMÍREZ, L. Caracterización productiva de un sistema de cosecha a tala rasa de árbol completo con procesador en rodales de Pinus radiata D. Don. 1997. 46 f. Tesis (Grado) - Facultad de Ciencias Forestales, Universidad de Talca, Talca, Chile, 1997.

RANDHAWA, S., SCOTT, T.; OLSEN, E. Timber harvester: A microcomputer-based system for automatic selection of timber harvesting equipment. Applied Engineering in Agriculture, v. 8, n. 1, p. 121 - 127, 1992.

WANG, J.; LEDOUX, C. Estimating and validating ground-based timber harvesting production through computer simulation. Forest Science, v. 49, n. 1, p. 64 - 76, 2003. 